\title{
Epidemiology of cystic fibrosis respiratory pathogens isolated at a South African Hospital, 2006-2010
}

\author{
Vindana Chibabhai ${ }^{\mathrm{a}, \mathrm{b} *}$ and Warren Lowman ${ }^{\mathrm{b}, \mathrm{c}, \mathrm{d}}$ \\ ${ }^{a}$ National Health Laboratory Service (NHLS), Department of Clinical Microbiology and Infectious Diseases, University of the Witwatersrand, \\ Johannesburg, South Africa \\ ${ }^{b}$ Department of Clinical Microbiology and Infectious Diseases, University of Witwatersrand, Johannesburg, South Africa \\ 'Wits Donald Gordon Medical Centre, Johannesburg, South Africa \\ ${ }^{d}$ Vermaak and Partners Pathologists, Pretoria, South Africa \\ *Corresponding author, emails: vindanac@yahoo.com, vindana.chibabhai@nhls.ac.za
}

Background: The epidemiology of cystic fibrosis (CF) associated pathogens other than Pseudomonas aeruginosa in the South African cystic fibrosis population has not been previously described.

Methods: A retrospective review of respiratory cultures taken from cystic fibrosis clinic patients at the Charlotte Maxeke Johannesburg Academic Hospital from 2006 to 2010 was performed.

Results: During the study period, Pseudomonas aeruginosa, Stenotrophomonas maltophilia, Burkholderia cepacia complex and Candida albicans prevalence remained stable, Aspergillus fumigatus increased from $8 \%$ to $20 \%$ ( $p=0.0132$ ); Staphylococcus aureus decreased from $66 \%$ to $50 \%(p=0.0243)$ and Haemophilus influenzae decreased from $13 \%$ to $3 \%(p=0.0136)$. There were significant antimicrobial susceptibility changes to meropenem $(p<0.0001)$ amongst $P$. aeruginosa isolates and cloxacillin $(p<0.0001)$ amongst $S$. aureus isolates. Prevalence of most bacterial pathogens appeared to increase with increasing age. Conclusion: The findings of this study illustrate the epidemiology of CF associated respiratory pathogens and the trends in prevalence and susceptibility patterns over a 5-year period.

Keywords: antimicrobial susceptibility, cystic fibrosis, Pseudomoas aeruginosa, respiratory pathogens, Staphyloccus aureus

\begin{abstract}
Introduction
Cystic fibrosis (CF) is a genetic disorder due to mutations in the CF transmembrane conductance regulator gene (CFTR), with functions in chloride ion transport. The gene mediates chloride ion conduction and activates chloride ion channel activity. ${ }^{1}$ CFTR mutations result in reduced chloride secretion into the airways and increased absorption of sodium from the airways. This results in a viscous airway and mucous that affects mucociliary clearance, resulting in persistent mucin secretion, mucous plug formation and persistent bacterial infections. ${ }^{2}$
\end{abstract}

CF patients may be infected with respiratory commensals or opportunistic pathogens. Commonly isolated organisms include Haemophilus influenzae, Staphylococcus aureus, Pseudomonas aeruginosa, Burkholderia cepacia complex, Stenotrophomonas maltophilia, Achromobacter xylosoxidans, Aspergillus fumigatus and non-tuberculous mycobacteria. ${ }^{3}$ Numerous reports have described the differing prevalence of these organisms within countries and between centres. ${ }^{4}$

South African CF statistics estimate that 1 in 3000 caucasians, 1 in 10300 individuals of mixed ancestry and up to 1 in 4624 black Africans carry a CFTR mutation. ${ }^{5}$ The aim of this study is to report on the prevalence of respiratory pathogens infecting the CF population, to describe age-specific trends and to document changes in antimicrobial resistance.

\section{Materials and methods}

A retrospective review of all respiratory cultures obtained from CF patients attending the CF clinics at Charlotte Maxeke Johannesburg Academic Hospital (CMJAH) was performed.
Respiratory cultures are routinely collected at clinic visits and do not always reflect acute episodes of infection.

\section{Study cohort}

The study cohort included patients who attended the CF clinics between 2006 and 2010, had a confirmed diagnosis of CF and a minimum of one respiratory culture specimen submitted to the National Health Laboratory Service Microbiology Laboratory for bacterial, fungal and/or mycobacterial microscopy, culture and susceptibility testing. The following information related to the patient was collected: date of birth, age and race. The following information related to respiratory samples submitted for culture was collected: date of the collection, specimen type, pathogens isolated and susceptibility to antimicrobials of interest.

\section{Microbiology methods}

A gram stain was performed on samples, which were then plated out onto $5 \%$ sheep blood agar; bacitracin-heated blood agar incubated at $35{ }^{\circ} \mathrm{C}$ in $5 \% \mathrm{CO}_{2}$; MacConkey agar; Pseudomonas cepacia agar; and, mannitol salt agar incubated aerobically at $35^{\circ} \mathrm{C}$. All plates were examined after 24 and $48 \mathrm{~h}$ of incubation. Specimens/isolates for fungal culture were plated out onto dextrose agar with chloromycetin and incubated aerobically at $35^{\circ} \mathrm{C}$ and $25^{\circ} \mathrm{C}$ for 14 days. A Periodic acid-Schiff and a Kinyoun stain were performed on samples for fungal culture. Identification and susceptibility testing of significant bacterial species was performed either by the MicroScan WalkAway automated identification/antimicrobial susceptibility testing (ID/AST) system (Siemens, Germany) or using an API (Analytical Profile Index-bioMérieux, France) and Kirby-Bauer susceptibility testing 
method. Identification of yeasts was performed by an API 20AUX (bioMérieux, France) and mould identification was performed by macroscopic and microscopic morphological examination of the isolate.

\section{Study definitions}

Prevalence was defined as the number of patients with a positive culture for a pathogen of interest during the study year. Only a single culture per patient was included in the analysis of prevalence. Prevalence of susceptibility to an antimicrobial was defined as the total number of isolates susceptible to an antimicrobial for a pathogen of interest during the study year. The age category in which a patient was considered was determined by age on 31 December of each year. Age categories included: $0-1$ year, 2-4 years, 5-9 years, $10-14$ years, 15-20 years and $\geq 21$ years.

Antimicrobial susceptibility definitions set by the Clinical Laboratory Standards Institute (CLSI) were used, ${ }^{6-10}$ and were determined by the Kirby-Bauer disk diffusion method or minimum inhibitory concentrations (MIC's) determined by the MicroScan WalkAway automated ID/AST system.

\section{Statistical analysis}

Annual and age specific prevalence were determined for $P$. aeruginosa, S. aureus, $H$. influenzae, $B$. cepacia complex, $S$. maltophilia, A. xylosoxidans, C. albicans and A. fumigatus. The 2-sample proportion test was used to determine the statistical significance of changes between 2006 and 2010. Prevalence of susceptibility to commonly used antimicrobials was determined for the majority of bacterial pathogens. Change in prevalence over the study period was determined for each pathogen, as was change in susceptibility to antimicrobials.

\section{Results}

One hundred and seventy-seven (177) patients were seen at the CMJAH cystic fibrosis clinics during 2006 and 2010. Twenty-two patients were excluded from the analysis: 4 were negative for $C F$ mutations and 18 had no respiratory samples submitted. Eightyfive patients were male, and 70 were female. The study cohort consisted of 130 caucasians, 13 black Africans, 5 patients of Indian origin, 8 of mixed ancestry and 1 patient whose race was unknown. The number of patients seen at the clinic per year ranged between 88 and 94 . The age of patients ranged between 2 months and 80 years of age (median 16 years).

\section{Annual prevalence of bacterial respiratory pathogens}

The annual prevalence of each pathogen is shown in Table 1. The most prevalent organism cultured was $P$. aeruginosa followed by S. aureus. H. influenzae, B. cepacia complex and S. maltophilia were less prevalent organisms with fewer than $15 \%$ of patients having positive cultures for these organisms. A. xylosoxidans was cultured in $\leq 2 \%$ of patients in 3 of the 5 years of the study period.

\section{Overall change in the prevalence of bacterial pathogens over the 5-year period}

Statistically significant changes in prevalence were observed for S. aureus with a reduction from $66 \%$ to $50 \%(p=0.0243)$. $H$. influenzae also demonstrated a reduction in prevalence from $13 \%$ to $3 \%(p=0.0136)$. Changes in prevalence of other bacterial pathogens were not statistically significant (Table 2 ).

\section{Age specific prevalence of bacterial pathogens}

$P$. aeruginosa and $S$. aureus demonstrated a gradual increase in prevalence with age. S. maltophilia was most prevalent in the 10-14-year age group, a finding described in the USA, ${ }^{11}$ whereas

Table 1: Yearly prevalence of bacterial pathogens

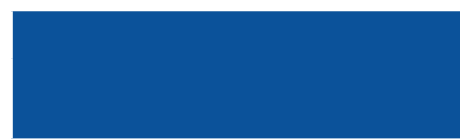

Total number of patients seen at the clinic per year

P. aeruginosa

S. aureus

H. influenzae

B. cepacia complex

S. maltophilia

A. xylosoxidans

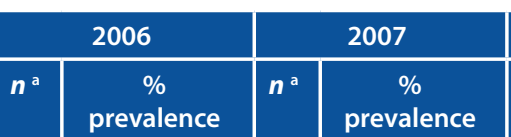

92

91

\begin{tabular}{|c|c|}
\multicolumn{2}{|c}{2008} \\
\hline$n^{\text {a }}$ & $\%$ \\
& prevalence
\end{tabular}

93

\begin{tabular}{|l|l|}
\hline 71 & $77 \%$ \\
\hline 61 & $66 \%$ \\
\hline 12 & $13 \%$ \\
\hline 6 & $7 \%$ \\
\hline 2 & $2 \%$ \\
\hline 2 & $2 \%$ \\
\hline
\end{tabular}

\begin{tabular}{|c|c|c|}
\hline 78 & $86 \%$ & 70 \\
\hline 61 & $67 \%$ & 61 \\
\hline 14 & $15 \%$ & 10 \\
\hline 4 & $4 \%$ \\
\hline 0 & $0 \%$ \\
\hline 1 & $1 \%$ \\
\hline
\end{tabular}

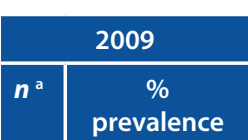

88

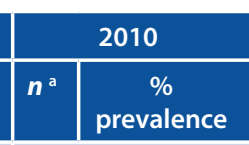

94

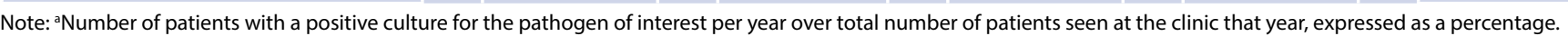

Table 2: Change in prevalence of bacterial pathogens between 2006 and 2010

\begin{tabular}{|l|c|c|c|c|}
\hline Organism & Prevalence in 2006 (\%) & Prevalence in 2010 (\%) & Change in Prevalence (\%) & p value \\
\hline P. aeruginosa & 77 & 85 & 0.1664 \\
\hline S. aureus & 66 & 50 & -16 \\
\hline H. influenzae & 13 & 3 & -10 \\
\hline B. cepacia complex & 7 & 11 & 0.0243 \\
\hline S. maltophilia & 2 & 4 & 0.0136 \\
\hline A. xylosoxidans & 2 & 1 & 0.4218 \\
\hline
\end{tabular}


Table 3: Changes to antimicrobial susceptibility among $P$. aeruginosa isolates

\begin{tabular}{|c|c|c|c|c|c|c|}
\hline Antibiotic & $\begin{array}{l}\text { No. Susceptible/ } \\
\text { total number } \\
\text { of isolates } \\
\text { with available } \\
\text { susceptibility } \\
\text { data } 2006\end{array}$ & $\begin{array}{l}\text { Percentage (\%) } \\
\text { susceptible } 2006\end{array}$ & $\begin{array}{l}\text { No. Susceptible/ } \\
\text { total number } \\
\text { of isolates } \\
\text { with available } \\
\text { susceptibility } \\
\text { data } 2010\end{array}$ & $\begin{array}{c}\text { Percentage (\%) } \\
\text { susceptible } 2010\end{array}$ & $\begin{array}{c}\text { Change in } \\
\text { susceptibility } \\
\text { between } 2006 \\
\text { and } 2010\end{array}$ & $p$ value \\
\hline Ceftazidime & $390 / 446$ & $87.4 \%$ & $226 / 266$ & $84.9 \%$ & $2.5 \%$ & 0.3483 \\
\hline Cefepime & $377 / 446$ & $84.52 \%$ & $225 / 266$ & $84.58 \%$ & $-0.06 \%$ & 0.9837 \\
\hline $\begin{array}{l}\text { Piperacillin- } \\
\text { Tazobactam }\end{array}$ & $401 / 446$ & $89.91 \%$ & $230 / 267$ & $86.14 \%$ & $3.77 \%$ & 0.1269 \\
\hline Meropenem & $422 / 446$ & $94.61 \%$ & $200 / 265$ & $75.47 \%$ & $19.14 \%$ & $<0.0001$ \\
\hline Imipenem & $385 / 446$ & $86.32 \%$ & $224 / 264$ & $84.84 \%$ & $1.52 \%$ & 0.5868 \\
\hline Ciprofloxacin & $367 / 446$ & $82.28 \%$ & $227 / 264$ & $85.98 \%$ & $-3.7 \%$ & 0.1977 \\
\hline Gentamicin & $329 / 446$ & $73.76 \%$ & $185 / 263$ & $70.34 \%$ & $3.42 \%$ & 0.3239 \\
\hline Amikacin & $352 / 446$ & $78.92 \%$ & $200 / 266$ & $75.18 \%$ & $3.74 \%$ & 0.2480 \\
\hline Tobramycin & $375 / 446$ & $84.08 \%$ & $227 / 263$ & $86.31 \%$ & $-2.23 \%$ & 0.4227 \\
\hline
\end{tabular}

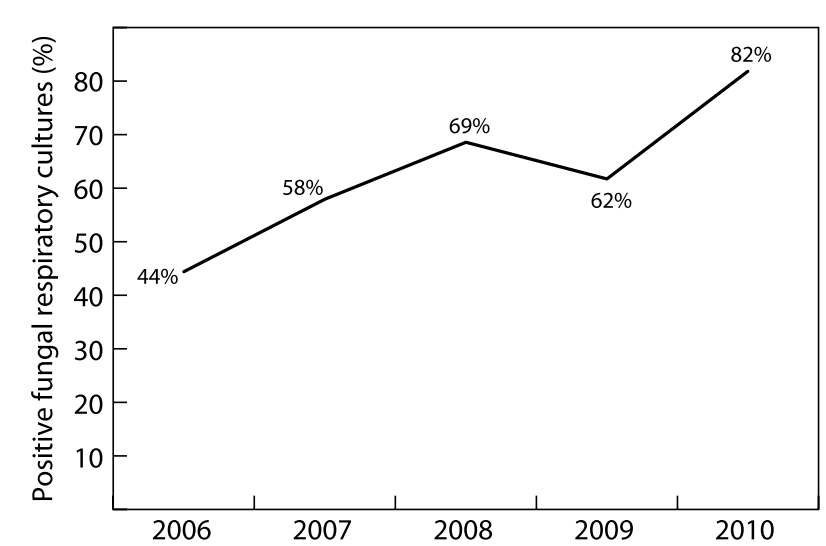

Figure 1: Respiratory fungal culture positivity rate.

$H$. influenzae was most prevalent in the 5-9 year age bracket. $B$. cepacia complex was only isolated once in a child $<10$ years of age. All other B. cepacia complex cultures were isolated from children $>10$ years and in adults. A. xylosoxidans was only isolated in children over 8 years of age and adolescents. The supplementary information provided illustrates the prevalence of each pathogen according to age category.

\section{Change in Susceptibility to Selected Antimicrobial Agents}

\section{P. aeruginosa}

$P$. aeruginosa isolates demonstrated no significant difference in susceptibility between 2006 and 2010 for the majority of antimicrobials tested. Only a markedly significant decrease in susceptibility was noted for meropenem from $94.61 \%$ to $75.47 \%$ $(p<0.0001)$ (Table 3).

\section{S. aureus}

Cloxacillin susceptibility among $S$. aureus isolates decreased significantly from $92 \%$ to $78 \%(p<0.0001)$ suggesting an increase in the rate of MRSA of $14 \%$ over the 5 -year period. There were no vancomycin intermediate or resistant S. aureus isolates detected during the study period. Trends in vancomycin MIC's were not analysed during this study and cannot be commented on.

\section{H. influenzae}

Ampicillin susceptibility (detected by $\beta$-lactamase production) amongst $H$. influenzae isolates decreased from $100 \%$ to $83 \%$ $(p=0.0687)$. No $\beta$-lactamase negative ampicillin resistant isolates of $H$. influenzae were isolated during the study period.

\section{S. maltophilia}

No change in susceptibility of S. maltophilia isolates was noted between 2006 and 2010. All isolates remained susceptible to trimethoprim-sulphamethoxazole.

\section{B. cepacia complex}

The susceptibility of $B$. cepacia complex isolates to trimethoprimsulphamethoxazole, ceftazidime and meropenem remained stable during the study period.

\section{Bacterial co-infections}

Co-infections involved predominantly P. aeruginosa and S. aureus isolates. Of $1774 P$. aeruginosa isolates, 324 were isolated with another pathogen. The predominant co-infecting pathogens were S. aureus $(81 \%)$ and B. cepacia complex (10\%). S. aureus isolates demonstrated the second highest likelihood of being cultured with another pathogen (370 of 1039). P. aeruginosa was the co-infecting pathogen in $71 \%$ of cases, and B. cepacia complex in $15 \%$ of cases. A total of 128 B. cepacia complex isolates were cultured during the study period, of which $93(73 \%)$ were isolated with another pathogen.

\section{Other Bacterial Pathogens}

Numerous other bacterial species were isolated during the study period. The four most commonly isolated bacteria included Enterobacter spp. (22 isolates), Klebsiella spp. (21), Escherichia coli (15) and Streptococcus pneumoniae (17).

\section{Fungal Pathogens}

During the 5-year study period, the number of positive fungal cultures increased from $44 \%$ to $82 \%(p<0.0001)$ (Figure 1). The 
Table 4: Yearly prevalence of the most commonly isolated yeast and mould species between 2006 and 2010
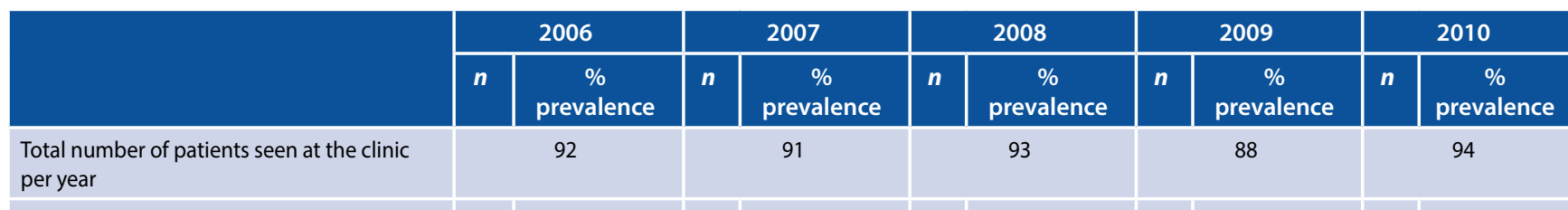

92

91

93

88

94

C. albicans

A. fumigatus

A. terreus

A. flavus

C. parapsilosis

C. dubliniensis

C. glabrata

Penicillium spp.

P. boydii

Scedosporium spp.

\begin{tabular}{|c|c|c|c|}
\hline 26 & $28 \%$ & 26 & $29 \%$ \\
\hline 7 & $8 \%$ & 7 & $8 \%$ \\
\hline 2 & $2 \%$ & 3 & $3 \%$ \\
\hline 0 & $0 \%$ & 3 & $3 \%$ \\
\hline 1 & $1 \%$ & 4 & $4 \%$ \\
\hline 0 & $0 \%$ & 1 & $1 \%$ \\
\hline 0 & $0 \%$ & 1 & $1 \%$ \\
\hline 2 & $2 \%$ & 1 & $1 \%$ \\
\hline 2 & $2 \%$ & 6 & $7 \%$ \\
\hline 2 & $2 \%$ & 0 & $0 \%$ \\
\hline
\end{tabular}

\begin{tabular}{|l|l|}
\hline \multicolumn{2}{|c|}{93} \\
\hline 29 & $31 \%$ \\
\hline 13 & $14 \%$ \\
\hline 4 & $4 \%$ \\
\hline 9 & $10 \%$ \\
\hline 3 & $3 \%$ \\
\hline 1 & $1 \%$ \\
\hline 2 & $2 \%$ \\
\hline 2 & $2 \%$ \\
\hline 2 & $2 \%$ \\
\hline 2 & $2 \%$ \\
\hline
\end{tabular}

\begin{tabular}{|l|l|}
\hline \multicolumn{2}{|c|}{88} \\
\hline 25 & $28 \%$ \\
\hline 10 & $11 \%$ \\
\hline 4 & $5 \%$ \\
\hline 6 & $7 \%$ \\
\hline 3 & $3 \%$ \\
\hline 2 & $2 \%$ \\
\hline 0 & $0 \%$ \\
\hline 2 & $2 \%$ \\
\hline 3 & $3 \%$ \\
\hline 2 & $2 \%$ \\
\hline
\end{tabular}

\begin{tabular}{|c|c|}
\hline \multicolumn{2}{|c|}{94} \\
\hline 34 & $36 \%$ \\
\hline 19 & $20 \%$ \\
\hline 4 & $4 \%$ \\
\hline 5 & $5 \%$ \\
\hline 0 & $0 \%$ \\
\hline 2 & $2 \%$ \\
\hline 2 & $2 \%$ \\
\hline 3 & $3 \%$ \\
\hline 4 & $4 \%$ \\
\hline 0 & $0 \%$ \\
\hline
\end{tabular}

prevalence of $C$. albicans remained stable during the 5 -year period ( $28 \%$ in 2006 to $36 \%$ in $2010 ; p=0.2486$ ); however, a significant increase in the prevalence of $A$. fumigatus from $8 \%$ to $20 \%$ ( $p=$ 0.0132 ) was observed. Of note, there was a significant increase in the prevalence of Aspergillus flavus from 0\% in 2006 to 5\% in 2010 $(p=0.03)$. A small number of non-albicans Candida spp. and other moulds were isolated during the study period. Table 4 lists the number of isolates and the respective prevalence of the most commonly isolated yeasts and moulds during the study period.

Figure 1 depicts the statistically significant increase $(p<0.001)$ in the number of samples which cultured fungal pathogens during each year of the study period.

\section{Mycobacteria}

A total of 133 samples were submitted for mycobacterial culture. Only 4 samples yielded mycobacteria. Mycobacterium tuberculosis complex was isolated from 2 samples. Both of these isolates were susceptible to rifampicin and isoniazid. One culture of Mycobacterium avium complex and one culture of Mycobacterium coronae were isolated. In addition, 2 specimens were smear positive for acid-fast bacilli, but contamination of the culture prevented identification. Forty-five percent (45\%) of mycobacterial culture samples were contaminated, suggesting that the prevalence of mycobacteria may have been underestimated.

\section{Discussion}

The findings from this study provide important epidemiological information regarding the prevalence of pathogens among South African CF patients. The study provides the opportunity to assess changes in epidemiology of these pathogens.

The average prevalence of each pathogen is listed in Table 5, and is compared to reported rates from other publications. From the table, the prevalence of $P$. aeruginosa in our study appears to be higher in comparison to other studies. The study by Pentz et al. ${ }^{12}$ describes the effect of chronic $P$. aeruginosa colonisation on lung function in a small South African cohort of cystic fibrosis patients. The reported prevalence is lower and may be due to the small number of patients included in the study. As mentioned earlier, different sites reporting on a demographically similar population group have reported dissimilar prevalences. The prevalence of $S$. maltophilia and A.xylosoxidans in our study appear comparatively lower. S. aureus prevalence in our study was similar to that described by Razvi et al. ${ }^{11}$. However, no direct comparison to these publications can be made, since the study periods as well as age ranges differ from that of our study.

Non-significant prevalence changes among P. aeruginosa, $S$. maltophilia and $B$. cepacia complex suggest stability of these established CF pathogens. The reasons for a decrease in S. aureus

Table 5: Comparison of the data from this study to other studies of this nature

\begin{tabular}{|l|c|c|c|c|c|c|}
\hline Organism & This study & Pentz et al. 2012 & $\begin{array}{c}\text { LiPuma (USA) } \\
\text { Based on CFF } \\
\text { Patient Registry } \\
\text { Data }\end{array}$ & $\begin{array}{c}\text { Valenza et al. } \\
\mathbf{2 0 0 7}^{13}\end{array}$ & $\begin{array}{c}\text { Lambiase et al. } \\
\mathbf{2 0 0 6}^{14}\end{array}$ & $\begin{array}{c}\text { Millar et al. 2009 } \\
\text { (2005 data) }\end{array}$ \\
\hline P. aeruginosa & $81 \%$ & $51 \%$ & $52.2 \%$ & $30 \%$ & $40 \%$ & $82 \%$ \\
\hline B. cepacia complex & $7 \%$ & NR & $3-4 \%$ & 0 & $7 \%$ & $4 \%$ \\
\hline S. aureus & $59 \%$ & NR & $50 \%$ & $38 \%$ & NR & $82 \%$ \\
\hline H. influenzae & $9 \%$ & NR & $10.3 \%$ & $10 \%$ & NR & $6 \%$ \\
\hline S. maltophilia & $2 \%$ & NR & $12 \%$ & $9 \%$ & $11 \%$ & $4 \%$ \\
\hline A. xylosoxidans & $1 \%$ & NR & $6 \%$ & NR & $7 \%$ & $2 \%$ \\
\hline
\end{tabular}

NR: Not Reported.

CFF: Cystic Fibrosis Foundation. 
and $H$. influenzae prevalence are unclear. There were no changes in laboratory isolation methods for either of these pathogens. There were no changes to the management of patients colonised with these pathogens according to the South African CF Consensus Document. ${ }^{16}$ However, as is stated in the document, prophylactic anti-staphylococcal agents may delay the development of chronic infection with S. aureus. At the same time, the suppression of $S$. aureus allows for early colonisation with $P$. aeruginosa, which may account for the high prevalence of P. aeruginosa. A South African publication describes high rates of invasive $H$. influenzae type b during the period from 2003 to 2009 despite high vaccine coverage rates (69\%), which remained fairly stable in comparison to previous data describing a $70 \%$ vaccine coverage rate in $2003 .{ }^{17}$ Although not directly comparable, the decreased prevalence in this study may be attributable to better vaccine coverage among the CF patients.

P. aeruginosa, S. aureus, and B. cepacia complex were more prevalent in older patients, as has been described in other studies. ${ }^{3}$ In this study population, B. cepacia complex was isolated almost exclusively in patients over 10 years of age with only one isolate from a 9-year-old boy. $\mathrm{H}$. influenzae was isolated most commonly in patients between $2-10$ years of age. ${ }^{18} \mathrm{~S}$. maltophilia was isolated in very small numbers overall: only 9 isolates during the 5 -year period. A recent publication has questioned the role of S. maltophilia as a pathogenic organism in the CF population. ${ }^{19}$

There was a significant change in susceptibility to meropenem among $P$. aeruginosa isolates. This change cannot be accounted for by changes in CLSI interpretive guidelines. There may have been extensive empiric use of meropenem since the guidelines were not specific about which intravenous antimicrobials to use in ill patients infected with $P$. aeruginosa. Also, the recommended duration of treatment is a minimum of 2 weeks and the ability of $P$. aeruginosa to develop resistance during antimicrobial therapy has been well described..$^{20} \mathrm{~A}$ marked and statistically significant decrease in methicillin susceptibility was noted among S. aureus isolates. These changes may be accounted for by the addition of more stringent CLSI interpretive breakpoints for cefoxitin in 2007. ${ }^{7}$ National $H$. influenzae antimicrobial surveillance data reported that $14 \%$ of non-typeable strains and $21 \%$ of type $b$ strains were ampicillin non-susceptible. ${ }^{21}$ These findings are consistent with our study findings. However, interpretation of the findings related to susceptibility profiles was limited by the lack of clinical data and data associated with antimicrobial usage. Also, there were no isolates displaying decreased susceptibility to polymyxins (colistin), despite a number of multidrug resistant $P$. aeruginosa isolates.

Co-infection among bacterial pathogens tend to occur most commonly between 3 organisms, viz P. aeruginosa, S. aureus and $B$. cepacia complex. Findings from previous studies have demonstrated an increase in biofilm formation when $P$. aeruginos $a$ and $B$. cenocepacia co-infect the CF lung, ${ }^{22}$ suggesting added difficulty in eradicating both pathogens with conventional antimicrobial therapy. The effect of co-infection with S. aureus or $B$. cepacia complex and $P$. aeruginosa has been shown to increase inflammatory markers in the lower respiratory tract, thus worsening clinical outcomes. . $2,23^{2}$

Enterobacteriaceae feature prominently as colonisers of the respiratory tract of CF patients. Publications have described Serratiamarcescens as the most common of the Enterobacteriaceae isolated from CF patients. ${ }^{24}$ Some have explored the possibility of
E. coli as an unrecognised CF-associated pathogen..$^{25}$ In addition, a number of S. pneumoniae isolates were cultured: a noteworthy pathogen cultured from CF patients due to the severity of disease it has the propensity to cause.

There was a statistically significant increase in the number of positive fungal cultures over the study period. This may reflect a true increase since laboratory isolation practices did not change during this time. This reported increase may also reflect an increased awareness amongst clinicians regarding the role of fungi in CF populations. Candida albicans was isolated frequently in this population. This may have been as a result of suboptimal specimen collection, resulting in contamination of respiratory samples with oral flora, or may represent colonisation of the respiratory tract following multiple courses of antibiotics. The quality of specimens was not taken into account when analysing the data, and therefore limits interpretation of the findings. A significant increase in prevalence of $A$. fumigatus between 2006 and 2010 may also suggest a true increase in prevalence of this pathogen. Interesting to note: the high prevalence of Aspergillus flavus, which is described elsewhere as a transient coloniser. ${ }^{26}$

Mycobacterial culture techniques are plagued with problems, particularly related to contamination. ${ }^{27}$ The rate of positive mycobacteria cultured during this study is likely to have been underestimated as a result of the high number of contaminated samples. Since 2010 however, there have been marked improvements in laboratory diagnostics, including molecular techniques applied directly to acid fast positive sputum samples, and it is likely that the reported prevalence in subsequent years may be higher.

This study has several limitations. Of particular note, colonisation versus infection was not assessed in this study. In some centres however, patients are treated at routine intervals irrespective of clinical signs of infection. When analysing the prevalence of susceptibility, the total number of samples submitted for culture per patient was not controlled for. This may have led to an overestimation or underestimation of resistance to certain antimicrobials. The absence of detailed clinical information is a further notable factor that limits the interpretation of the data. The findings of this study cannot be generalised outside of South Africa, and a countrywide study would be required to determine if the findings are similar at other CF centres. B. cepacia complex isolates were not further sub-speciated, thus it is not known what the distribution of isolates was within the complex. $B$. cenocepacia and $B$. multivorans are most likely associated with chronic infection, ${ }^{28}$ but chronic infection has been described with some of the other species in the complex, ${ }^{29}$ thus this additional knowledge would not have assisted with decisions regarding management or lung transplantation. However, speciation would have assisted in determination of potential clonality among the strains isolated. $H$. influenzae isolates were not typed, however this information would not have assisted with patient management. The lack of information regarding antimicrobial use, frequency of antibiotic courses, the route of administration, length of treatment and the effects of quorum sensing agents, such as azithromycin, limit clinical interpretation of the data presented.

\section{Conclusion}

This study describes the epidemiology of respiratory pathogens cultured from CF patients in South Africa. The typically described CF pathogens predominate in the South African population as; 
however, the prevalence of each pathogen differs somewhat to that described elsewhere. During the 5-year period, some changes in prevalence and antimicrobial susceptibility were noted. These changes need to be monitored continuously in order to regularly update empiric antimicrobial regimens in these patients. This study also emphasises the importance of a local CF culture database. CF patients are at high risk of developing severe disease and complications of respiratory tract infections, thus real-time monitoring of trends and changes in prevalence and susceptibility patterns will allow for prompt changes to treatment regimens to improve patient care.

Acknowledgments - Dr. Susan Klugman (CMJAH Paediatric CF Clinic) and Prof. Mervyn Mer (CMJAH Adult CF Clinic) for assistance with the acquisition of the CF Consensus Document and permitting use of the CF clinic during data collection.

Funding - No specific funding was available for this study.

Competing interests - All authors declare no conflict of interest.

\section{Ethical approval}

Obtained from the University of Witwatersrand Human Ethics Committee.

\section{Note}

Data from this manuscript was previously presented at the FIDDSA Congress 2013, entitled "Epidemiology and Prevalence of Respiratory Pathogens cultured from Cystic Fibrosis Patients attending the CMJAH Cystic Fibrosis Clinics during 2010" by Vindana Chibabhai and Warren Lowman.

\section{Supplementary material}

Supplementary material for this article can be accessed here http://dx.doi.org/10.1080/23120053.2016.1156864

\section{References}

1. Dorwart M, Thibodeau P, Thomas P. Cystic fibrosis: recent structural insights. J Cyst Fibros. 2004;3:91-94.

2. Boucher RC. An overview of the pathogenesis of cystic fibrosis lung disease. Adv Drug Deliv Rev. 2002;54:1359-1371.

3. LiPuma J. The changing microbial epidemiology in cystic fibrosis. Clin Microbiol Rev. 2010;23:299-323.

4. de Vrankrijker AM, Wolfs TFW, van der Ent CK. Challenging and emerging pathogens in cystic fibrosis. Paediatr Respir Rev. 2010;11:246-254.

5. Westwood T, Henderson B, Ramsay M. Diagnosing cystic fibrosis in South Africa. S Afr Med J. 2006 April;96(4):304.

6. CLSI M100-S16. Vol. 26 No.3. Performance Standards for Antimicrobial Susceptibility Testing; Seventeenth Information Supplement.

7. CLSI M100-S17. Vol. 27 No.1. Performance Standards for Antimicrobial Susceptibility Testing; Seventeenth Information Supplement.

8. CLSI M100-S18. Vol. 28 No.1. Performance Standards for Antimicrobial Susceptibility Testing; Seventeenth Information Supplement.

9. CLSI M100-S19. Vol. 29 No.3. Performance Standards for Antimicrobial Susceptibility Testing; Seventeenth Information Supplement.

10. CLSI M100-S20. Vol. 30 No.1. Performance Standards for Antimicrobial Susceptibility Testing; Seventeenth Information Supplement.
11. Razvi S, Quittell L, Sewall A, et al. Respiratory microbiology of patients with cystic fibrosis in the United States, 1995 to 2005. Chest. 2009;136:1554-1560.

12. Pentz A, Becker P, Masekela R, et al.The impact of chronic pseudomonal infection on pulmonary function testing in individuals with cystic fibrosis in Pretoria, South Africa. S Afr Med J. 2014;104(3):191-194.

13. Valenza G, Tappe $D$, Turnwald $D$, et al. Prevalence and antimicrobial susceptibility of microorganisms isolated from sputa of patients with cystic fibrosis. J Cyst Fibros. 2008;7:123-127.

14. Lambiase A, Raia V, Pezzo M, et al. Microbiology of airway disease in a cohort of patients with cystic fibrosis. BMC Infect Dis. 2006;6(1):4.

15. Millar FA, Simmonds NJ, Hodson ME. Trends in pathogens colonising the respiratory tract of adult patients with cystic fibrosis, 1985-2005 J Cyst Fibros. 2009;8:386-391.

16. South African Cystic Fibrosis Consensus Group. The South African Cystic Fibrosis Consensus Document. 4th ed. South African Cystic Fibrosis Association: Johannesburg; 2012.

17. von Gottberg A, Cohen C, Whitelaw A, et al. Invasive disease due to Haemophilus influenzae serotype b ten years after routine vaccination, South Africa, 2003-2009. Vaccine. 2012;30:565-571.

18. Cystic Fibrosis Foundation Patient Registry. Annual Data Report. MD: Bethesda; 2008.

19. Hansen C. Stenotrophomonas maltophilia. Curr Opin Pulmonary Med. 2012;18:628-631.

20. Lister PD, Wolter DJ, Hanson ND. Antibacterial-resistant Pseudomonas aeruginosa: Clinical impact and complex regulation of chromosomally encoded resistance mechanisms. Clin Microbiol Rev. 2009:22:582610.

21. Fortuin-de Smidt MC. Trends and Factors associated with invasive non-typeable and serotype b Haemophilus influenza disease in persons of all ages in South Africa from 2003-2009. Department of Public Health, Master's Degree Dissertation Report, University of the Witwatersrand: Johannesburg; 2013.

22. Bragonzi A, Farulla I, Paroni M, et al. Modelling co-infection of the cystic fibrosis lung by Pseudomonas aeruginosa and Burkholderia cenocepacia reveals influences on biofilm formation and host response. PLoS ONE. 2012;7:e52330.

23. Sagel SD, Gibson RL, Emerson J, et al. Impact of pseudomonas and staphylococcus infection on inflammation and clinical status in young children with cystic fibrosis. J Paediatr. 2009;154:183-188.e3.

24. Coenye T, Goris J, Spilker T, et al. Characterization of unusual bacteria isolated from respiratory secretions of cystic fibrosis patients and description of Inquilinus limosus gen. nov., sp. nov. J Clin Microbiol. 2002:40:2062-2069.

25. Barillova $P$, Tchesnokova $V$, Dübbers $A$, et al. Prevalence and persistence of Escherichia coli in the airways of cystic fibrosis patients-An unrecognized CF pathogen? Int J Med Microbiol. 2014;304(3-4):415-421.

26. Pihet M, Carrere J, Cimon B, et al. Occurrence and relevance of filamentous fungi in respiratory secretions of patients with cystic fibrosis- a review. Med Mycol. 2009;47(4):387-397.

27. Jarzembowski JA, Young, MB. Nontuberculous mycobacterial infections. Arch Pathol Lab Med. 2008;132:1333-1341.

28. Ciofu O, Hansen CR, Høiby N. Respiratory bacterial infections in cystic fibrosis. Curr Opin Pulmonary Med. 2013;19:251-258.

29. Boussaud V, Guillemain R, Grenet D, et al. Clinical outcome following lung transplantation in patients with cystic fibrosis colonised with Burkholderia cepacia complex: results from two French centres. Thorax. 2008;63:732-737.

Received: 27-07-2015 Accepted: 26-11-2015 